\title{
Enhanced vesicular stomatitis virus (VSV $\Delta 51)$ targeting of head and neck cancer in combination with radiation therapy or ZD6126 vascular disrupting agent
}

\author{
Nehad M Alajez ${ }^{1,2}$, Joseph D Mocanu ${ }^{2,3}$, Tiffany Krushel ${ }^{2,3}$, John C Bell ${ }^{4,5}$ and Fei-Fei Liü $2,3,6,7,8^{*}$
}

\begin{abstract}
Background: Head and neck squamous cell carcinoma (HNSCC) is the 5th most common cancer worldwide. Locally advanced HNSCC are treated with either radiation or chemo-radiotherapy, but still associated with high mortality rate, underscoring the need to develop novel therapies. Oncolytic viruses have been garnering increasing interest as anti-cancer agents due to their preferential killing of transformed cells. In this study, we evaluated the therapeutic potential of mutant vesicular stomatitis virus (VSVA51) against the human hypopharyngeal FaDu tumour model in vitro and in vivo.

Results: Our data demonstrated high toxicity of the virus against FaDu cells in vitro, which was associated with induction of apoptosis. In vivo, systemic injection of $1 \times 10^{9} \mathrm{pfu}$ had minimal effect on tumour growth; however, when combined with two doses of ionizing radiation (IR; 5 Gy each) or a single injection of the vascular disrupting agent (ZD6126), the virus exhibited profound suppression of tumour growth, which translated to a prolonged survival in the treated mice. Concordantly, VSVA51 combined with ZD6126 led to a significant increase in viral replication in these tumours.
\end{abstract}

Conclusions: Our data suggest that the combinations of VSVA51 with either IR or ZD6126 are potentially novel therapeutic opportunities for HNSCC.

Keywords: Oncolytic virus, HNSCC, Radiotherapy, Vascular disrupting

\section{Background}

Head and neck squamous cell carcinoma (HNSCC) is the most common cancer type in the head and neck region, accounting for the 5th most common cancer worldwide [1]. Locally advanced diseases are treated with either radiation or chemo-radiotherapy, but are still associated with $>50 \%$ mortality rate $[1,2]$, underscoring the need to develop novel therapeutic strategies. Oncolytic viruses have recently garnered increasing interest as anti-cancer agents due to their preferential killing of transformed cells (reviewed in [3,4]). Among these, the

\footnotetext{
* Correspondence: Fei-Fei.Liu@rmp.uhn.on.ca

${ }^{2}$ Ontario Cancer Institute, Toronto, ON, Canada

${ }^{3}$ Department of Medical Biophysics, University of Toronto, Toronto, ON,

Canada

Full list of author information is available at the end of the article
}

mutant variant of vesicular stomatitis virus (VSV) has been evaluated in different tumour models, demonstrating promising results as either single agent or in combination with other treatment modalities [5-7]. We had previously demonstrated the exquisite sensitivity of EBV-positive nasopharyngeal carcinoma (NPC) to a mutant VSV (VSV $\Delta 51$ ), which has a single amino acid deletion in the VSV M protein, rendering lethality to cancer cells, whilst sparing normal cells [8]. Building upon this observation, herein we evaluated the efficacy of VSV $\Delta 51$ against the human FaDu hypopharyngeal squamous cell carcinoma model either as a single agent, or combined with radiation therapy (RT) or the vascular disrupting agent ZD6126. ZD6126 is a colchicine prodrug derivative that is metabolized in vivo to yield ZD6126 phenol, which then selectively binds to the colchicine-binding

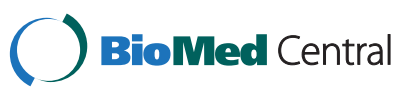


site of tubulin; this disrupts the microtubule structure, largely responsible for the structure and morphology of dividing and immature vascular endothelial cells [9]. Our data demonstrated enhanced efficacy of VSV 551 when combined with either RT or ZD6126 in vivo, thereby supporting the potential clinical utility of these combination strategies for head and neck cancer.

\section{Results}

\section{VSV $\Delta 51$ is lethal to FaDu cells in vitro}

To evaluate sensitivity of HNSCC cells to VSV $\Delta 51$, $\mathrm{FaDu}$ cells were infected with VSV $\Delta 51$ at $0.1 \mathrm{pfu} / \mathrm{cell}$.
Figure 1A demonstrated significant toxicity of VSV $\Delta 51$ against $\mathrm{FaDu}$ cell at $72 \mathrm{hrs}$ post infection (pi). Using the MTS cell viability assay, VSV $\Delta 51$ exhibited both dose-and time-dependent toxicity against $\mathrm{FaDu}$ cells (Figure 1B, left panel), which did not change significantly with the addition of 6 Gy ionizing radiation (IR, Figure $1 \mathrm{~B}$, right panel). Using cell cycle analysis, a significant proportion of cells underwent apoptotic cell death at $72 \mathrm{hrs}$ pi with $0.01 \mathrm{pfu} / \mathrm{cell}$ (Figure 1C, left panel). Combining VSV $\Delta 51$ with 4 Gy IR led to a slight increase in apoptosis (26\% vs. $22 \%)$. Similarly, induction of caspase 3 activation was observed in VSV $\Delta 51$ infected

A

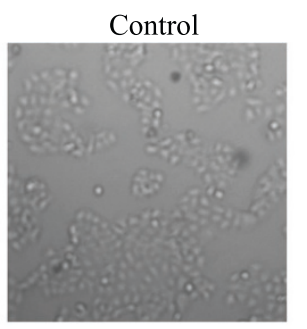

VSV-GFP
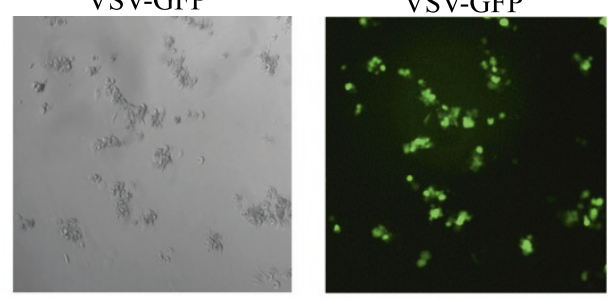

$\mathrm{B}$

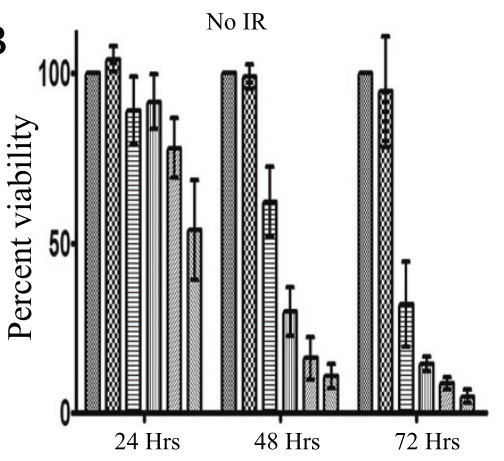

$\mathrm{C}$

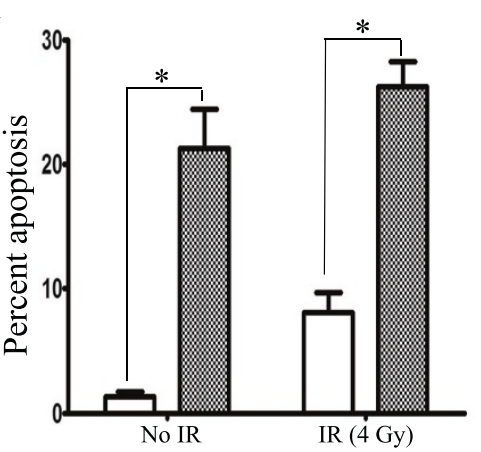

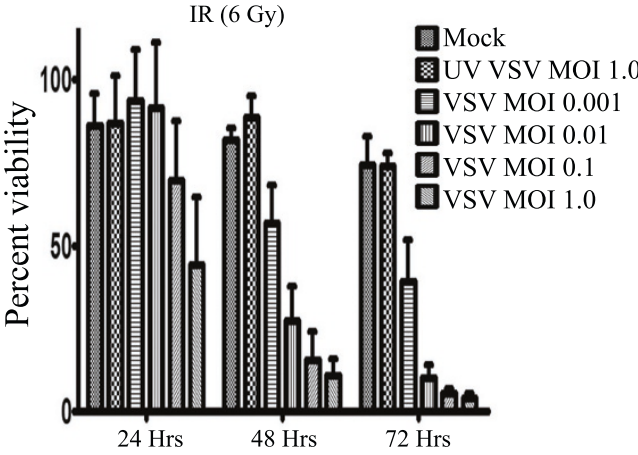

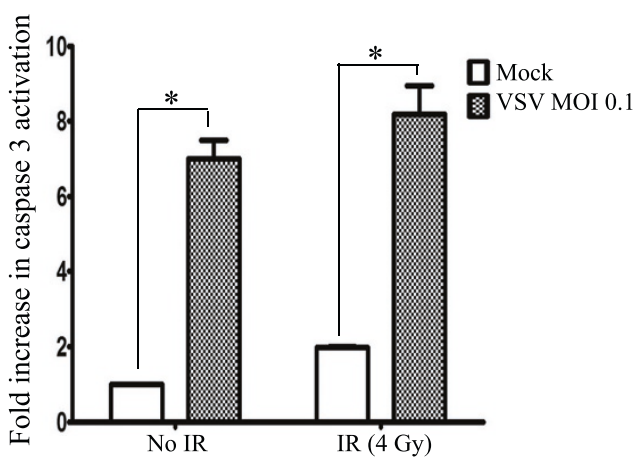

Figure 1 VSV $\Delta \mathbf{5 1}$ is lethal to FaDu cells in vitro. (A) FaDu Cells were visualized at 72 hrs post-infection with VSV $\Delta 51$ (0.1 pfu/cell) using light (middle) or fluorescence microscope (right) and compared to un-infected normal FaDu cells (left). (B) MTS assay for FaDu cell viability at 24, 48, and 72 hrs post-infection with the indicated doses of VSV 551 alone (left) or combined with 6 Gy IR (right) delivered just before infection. Data are presented as mean \pm SD from representative experiments, $n=3$. (C) Percent apoptosis (left panel) and fold increase in caspase 3 activity (right panel) in FaDu cells at 72 hrs post infection with 0.1 pfu/cell VSV $\Delta 51$. Data are presented as mean $\pm S E ; n=3$ from two independent experiments. ${ }^{*} p \leq 0.05$. 
cells, thus confirming apoptosis as a mode of cell death in infected cells (Figure 1C, right panel).

\section{Efficacy of VSV $\Delta 51$ combined with radiation therapy (RT) or ZD6126 in established FaDu xenograft model}

To determine whether the observed in vitro toxicity of VSV 551 against FaDu cell could be recapitulated in vivo, $\mathrm{FaDu}$ cells were implanted intramuscularly (im) into the left leg of CD-1 nude mice. Once the leg plus tumour diameter reached $\sim 9 \mathrm{~mm}$, the mice were randomized to: a) UV-inactivated VSV $\Delta 51$; (b) local tumour RTx2 (5 Gy each), 3 days apart; (c) VSV $\Delta 51$ alone; (d) RT (5 Gy) plus VSV $\Delta 51$ simultaneously; then $2^{\text {nd }} R T$ (5 Gy) 3 days later; (e) ZD6126 alone; and (f) ZD6126 just preceding VSV $\Delta 51$ injection. VSV $\Delta 51$ alone had modest effects, whereas two doses of 5 Gy RT led to a significant reduction in tumour growth (Figure 2A). When combined with RT, VSV $\Delta 51$ was able to significantly further decrease tumour growth compared to RT alone. Interestingly, the vascular disrupting agent ZD6126 had minimal effect on tumour growth when administered alone, but when combined with a single dose of VSV 551 , a significant inhibition of tumour growth was observed compared to either ZD6126 or VSV $\Delta 51$ alone (Figure 2A). Although the combination of VSV $\Delta 51$ with either RT or ZD6126 was not sufficient to completely eradicate the tumor, these combination led to the longest survival of tumour-bearing mice in that the median survival increased from $\sim 22$ (for the VSV alone group) to 48 and 47 days, respectively (Figure 2B). While no obvious increase in viral replication was observed in the RT plus VSV $\Delta 51$ group, images of tumour sections removed from treated mice revealed a significant increase in VSV $\Delta 51$ replication in the ZD6126 plus VSVA51-treated group, suggesting that the observed anti-tumour efficacy is likely attributable to enhanced viral deposition and replication in this group of mice (Figure 3).

\section{Discussion}

In recent years, oncolytic viruses have emerged as promising anti-cancer agents due to their demonstrated efficacy in a number of preclinical models [10-13], exerting a myriad of anti-tumour mechanisms ranging from direct tumour cell lysis, induction of apoptosis, to targeting of the tumour vasculature $[8,14,15]$. Despite these promising pre-clinical data, clinical trials utilizing oncolytic viruses have demonstrated limited success; accentuating the need for improvement. To that end, several studies have administered oncolytic viruses in conjunction with standard or targeted therapies, demonstrating enhanced anti-tumour efficacy [16,17]. Our group has previously reported that increased tumour interstitial fluid pressure (IFP) could hinder therapeutic efficacy of adenoviral gene therapy in vivo [18]. As a follow-up, we also observed that the vascular disrupting agent ZD6126 could enhance bio-distribution of anti-sense oligonucleotides in xenografttumours, mediated via reducing tumour IFP [19]. These two observations were hence combined, to evaluate whether anti-tumour efficacy of VSV $\Delta 51$ could be improved with the addition of ZD6126. It was gratifying to observe that the combination of VSVA51 with

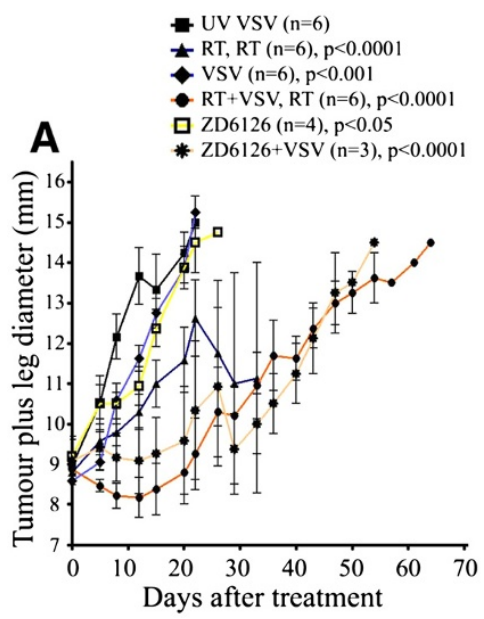

B

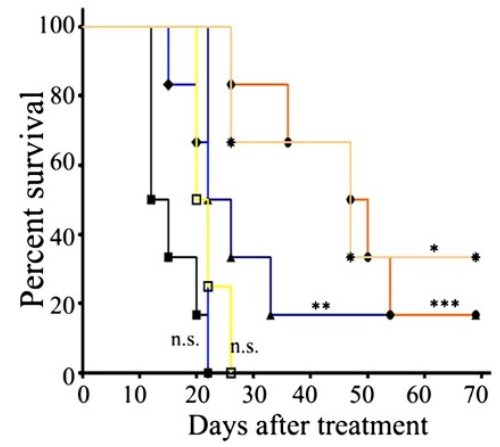

Figure 2 Efficacy of VSV $\mathbf{5 1}$ combined with RT or ZD6126 in the FaDu xenograft model. (A) FaDu xenograft tumours were established im in the left leg of CD-1 nude mice to allow for local RT delivery. Mice were then randomized to six groups: a) UV-inactivated VSV 551 $\left(1 \times 10^{9}\right.$ pfu i.v.); (b) local tumour RTX2 (5 Gy), 3 days apart; (c) VSV $551\left(1 \times 10^{9}\right.$ pfu i.v.); (d) RT (5 Gy each) plus VSV $\Delta 51\left(1 \times 10^{9}\right.$ pfu i.v.) simultaneously, then $2^{\text {nd }}$ RT (5 Gy) delivered 3 days later; (e) ZD6126 alone (5 mg i.v. in $200 \mu$ l); and (f) ZD6126 (5 mg) just preceding $1 \times 10^{9}$ VSV $\Delta 51$ i.v. Data are presented as mean leg plus tumour diameter \pm S.E. (B) Mice in panel (A) were monitored for survival for up to 70 days. All p-values were calculated using the Two-Way ANOVA analysis in comparison with the UV-inactivated VSV control group; survival comparisons were conducted using the log-rank test. n.S., not significant, ${ }^{*} p \leq 0.05,{ }^{* *} p \leq 0.005,{ }^{* * *} p \leq 0.0005$. 

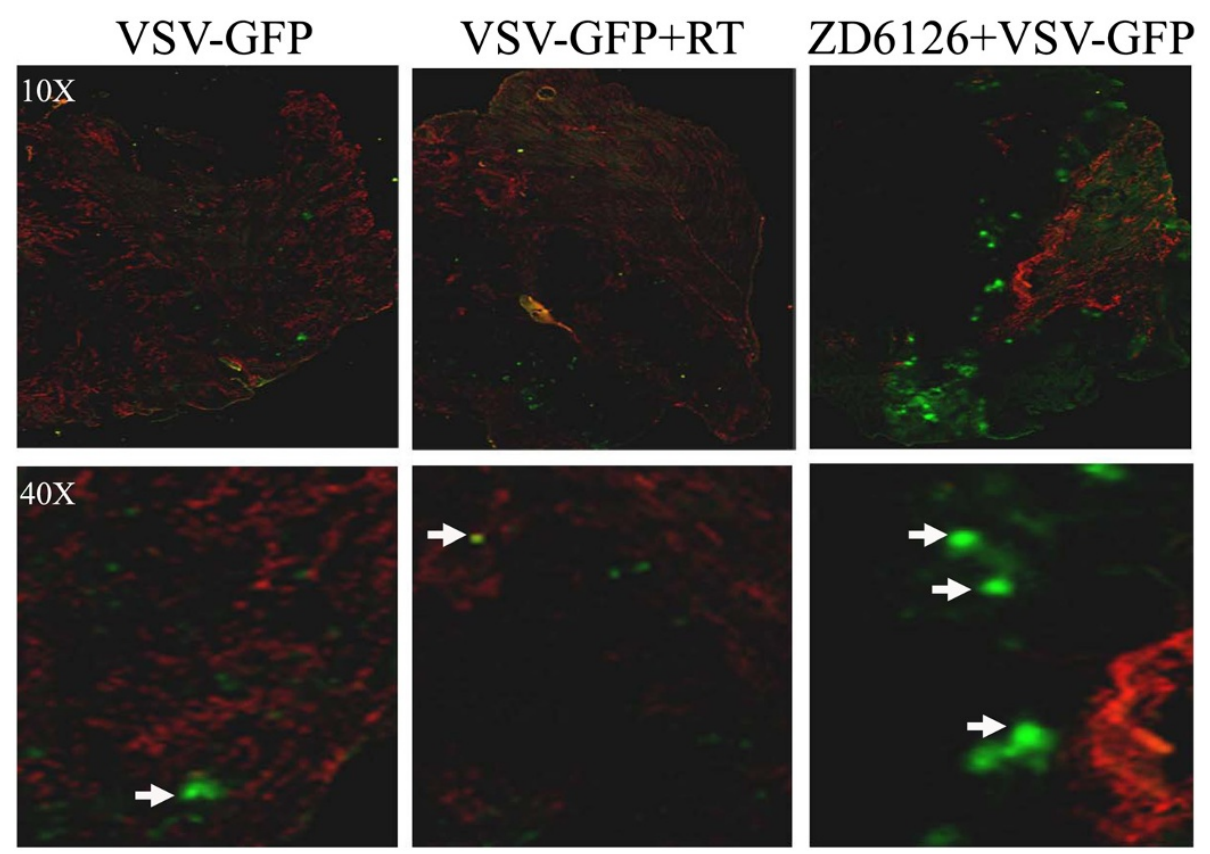

Figure 3 Representative examples of VSV $\mathbf{5} 51$ tumour microdistribution in FaDu xenograft tumours. Viral distribution (GFP) was assessed at $24 \mathrm{~h}$ post infection in relation to active vasculature (Hoechst, red). The merged field consisted of superimposed VSV, and Hoechst; the close-up images are high-resolution representations of GFP distribution in relation to local blood vessels.

ZD6126 was indeed effective in suppressing tumour growth, to a similar extent as that observed when VSV $\Delta 51$ was delivered in conjunction with local tumour RT (Figure 2A and B). Furthermore, enhanced viral presence was observed in the tumours treated with VSV $\Delta 51$ plus ZD6126 (Figure 3). The mechanisms could relate to both increased viral delivery consequent to tumour vasculature disruption leading to increased leakiness, plus reduced tumour IFP, both resulting in increased deposition and replication of the oncolytic virus.

When VSVA51 was combined with local tumour RT, enhanced viral replication was not observed, although effective anti-tumour activity was still apparent (Figures 2 \& 3). The extent of neutrophil infiltration was no different between the tumours treated with VSV $\Delta 51$ alone, or when combined with RT (data not shown), indicating that innate immunity cannot explain these results of the combination therapy. Hence, the mechanism of the additional tumour suppressive effect of VSV $\Delta 51$ combined with RT is likely an additive interaction between the cytotoxicity of the viral oncolytic therapy, plus the direct tumoricidal effects of ionizing radiation.

\section{Conclusions}

Enhanced efficacy of VSVA51 in an HNSCC tumour model is documented when combined with either local tumour RT, or with the use of a vascular disrupting agent ZD2612 in vivo. These combinational regimens have disparate mechanisms of anti-tumour efficacy, and have the potential as novel therapeutic strategies in the management of HNSCC.

\section{Methods}

\section{Ethics statement}

All animal experiments were conducted in accordance with the guidelines of the Animal Care Committee, University Health Network, Toronto, Canada.

\section{Cell lines and reagents}

$\mathrm{FaDu}$ human hypopharyngeal carcinoma cell line was purchased from the American Type Culture Collection (ATCC, Manassas, VA), and was authenticated at the Centre for Applied Genomics (the Hospital for Sick Children, Toronto, Canada) using the AmpF/STR Identifiler PCR Amplification Kit (Applied BiosystemsInc, Foster City, CA). Cells were cultured in in MEM-F15 supplemented with $10 \%$ fetal bovine serum (FBS), $100 \mathrm{mg} / \mathrm{L}$ penicillin, and $100 \mathrm{mg} / \mathrm{L}$ streptomycin (RPMI10) at $37^{\circ} \mathrm{C}, 5 \% \mathrm{CO}_{2}$. $293-\mathrm{F}$ Cells were purchased from invitrogen (Invitrogen, Carlsbad, CA).

\section{Virus production and cell infection}

VSV $\Delta 51$ carrying GFP (referred to as VSV $\Delta 51$ in this paper) was propagated in 293-F cells as previously described [8]. Briefly, 293-F cells were cultured in 293 SFM II medium (Invitrogen, Carlsbad, CA) in $500 \mathrm{~mL}$ 
spin-culture flasks, then were infected with VSV $\Delta 51$ GFP at MOI 0.01 in the presence of $1.8 \mathrm{mM} \mathrm{CaCl}_{2}$. Supernatant was collected 16-18 hours later, filtered through a $0.22 \mu \mathrm{m}$ filter, then collected by centrifugation at $18,500 \mathrm{~g}$ for $2.5 \mathrm{hrs}$ in a Sorvall RC5 centrifuge. UV inactivation was performed by exposing the virus for $30 \mathrm{~min}$ at $10 \mathrm{~cm}$ distance under UV light in a class II biosafety cabinet. For cell infection, 10,000 FaDu cells were seeded in $100 \mu \mathrm{L}$ of MEM-F15 plus $10 \%$ FBS in a 96-well plate. Twenty-four hrs later, cells were exposed to 0 or 6 Gy IR, medium was removed, and VSV $\Delta 51$ (MOI $=1.0-0.001)$ was added in $20 \mu \mathrm{L} \alpha$-MEM medium. The plates were kept at $37^{\circ} \mathrm{C}$ for 60 min to allow virus attachment prior to the addition of $80 \mu \mathrm{l}$ of normal growth medium (MEM-F15 plus 10\% FBS).

\section{Cell viability and measurement of apoptosis}

Cell viability was assessed using the MTS assay as previously described [20], and according to the manufacturerrecommended protocol (Promega, Madison, WI). Briefly, $20 \mu \mathrm{L}$ of the MTS reagent was added to each well in a 96-well plate at the indicated time points, and absorbance was read at $\lambda_{492}$. In order to measure the fraction of cells in the subG0/G1 phase of cell cycle, FaDu cells were infected with VSV $\Delta 51$ at MOI 0.1 ; at indicated time points, cells were harvested, and then washed twice in FACS buffer (PBS/0.5\% BSA). Cells were resuspended in $1 \mathrm{~mL}$ of FACS buffer, then $3 \mathrm{~mL}$ of icecold $70 \%$ ethanol was added to fix the cells for $1 \mathrm{hr}$ on ice. Cells were washed once, before re-suspending in 500 $\mu \mathrm{L}$ of FACS buffer supplemented with $40 \mu \mathrm{g} / \mathrm{mL}$ RNAseA (Sigma) and $50 \mu \mathrm{g} / \mathrm{mL}$ PI. Cells were incubated at room temperature for $30 \mathrm{~min}$ in the dark before being analyzed in the BD FACScalibur using FL-2A and FL$2 \mathrm{~W}$ channels. CaspGlow kit (Biovision, Mountain view, CA) was used to measure caspase 3 activity in virallyinfected $\mathrm{FaDu}$ cells. Briefly, infected cells were resuspended in $300 \mu \mathrm{L}$ of RPMI-10 followed by the addition of $1 \mu \mathrm{L}$ of FITC-labeled DEVD-FMK reagent for $45 \mathrm{~min}$ at $37^{\circ} \mathrm{C}$ to measure activated caspase 3. Following 45 mins' incubation, cells were washed, resuspended in buffer, then analyzed in BD FACScalibur using FL-1 channel.

\section{Animal experiments}

Five to six week old CD-1 nude male mice were purchased from Charles River Laboratories (Montreal, Canada); all experiments were conducted in accordance with the guidelines of the Animal Care Committee, University Health Network. For therapeutic experiments, $3 \times 10^{6} \mathrm{FaDu}$ cells were injected intramuscular (im) into the left leg. When tumour plus leg diameter reached 8.5$9.5 \mathrm{~mm}$, treatment was initiated. Mice were treated as indicated by injecting $1 \times 10^{9}$ pfu VSV $\Delta 51$ in $100 \mu \mathrm{L}$ PBS intravenously (iv), via the tail vein. For local radiation treatments, mice were immobilized in a Lucite box, and the tumor-bearing leg was exposed to $225 \mathrm{kVp}(13 \mathrm{~mA})$ at a dose rate of $3.37 \mathrm{~Gy} / \mathrm{min}$ using an X-ray irradiator C (X-RAD 225; Precision X-ray). ZD6126 (AstraZeneca) treatment was delivered as previously described [18]. Briefly, ZD6126 was dissolved in 10\% sodium carbonate and $90 \%$ PBS (pH 7.4, $25 \mathrm{mg} / \mathrm{mL}$ ). A single $200 \mu \mathrm{L}$ injection of ZD6126 solution was injected i.p. into $\mathrm{FaDu}-$ bearing nude mice. For the combined treatment, mice were treated with ZD6126 and then immediately injected with $1 \times 10^{9}$ pfu VSV $\Delta 51$ in $100 \mu \mathrm{L}$ PBS intravenously (iv), via the tail vein. Tumor growth was monitored by measuring tumor plus leg diameter as described before [21].

\section{Microdistribution studies}

$\mathrm{FaDu}$ xenograft tumours were established and treated as described above. At 24 hrs post-VSV injection, Hoechst $33342(600 \mu \mathrm{g}$ in $100 \mu \mathrm{L}$ PBS) was injected iv for visualization of the active vasculature [22]; the mice were sacrificed $1 \mathrm{~min}$ later. Tumours were immediately excised, frozen in Optimal Cutting Temperature (OCT) compound (Bayer Corporation, USA), then stored at $-80^{\circ} \mathrm{C}$. Serial sections (5 $\mu \mathrm{m}$ thickness) were cut through each tumour at three levels, $500 \mu \mathrm{m}$ apart. The slides were scanned using fluorescence microscopy to visualize GFP-expression and Hoechst 33342 perfusion. All staining and cryosectioning procedures were performed by Pathology Research Program Services, University Health Network (Toronto, Canada).

\section{Microscopy}

Slides were imaged at 10X magnification using an Olympus BX50 (Olympus, Japan) tiling fluorescence stereomicroscope (FITC/GFP: $\lambda_{\mathrm{Ex}}=482 \mathrm{~nm}, \lambda_{\mathrm{Em}}=535 \mathrm{~nm}, 500 \mathrm{~ms}$ exposure; Hoechst 33342: $\lambda_{\mathrm{Ex}}=360 \mathrm{~nm}, \lambda_{\mathrm{Em}}=460 \mathrm{~nm}$, $100 \mathrm{~ms}$ exposure). Images were captured using ImagePro 5.1 (Media Cybernetics, USA). Fluorescence microscopy images were imported into Adobe Photoshop and merged into a composite image, where the red, and green channels corresponded to Hoechst 33342 (active vasculature), and GFP (VSV infection), respectively.

\section{Statistical analysis}

Statistical analysis and graphing were performed using Microsoft Excel 2010, and Graphpad Prism 5.0 software.

\section{Competing interests}

There are no competing interests.

\section{Authors' contributions}

NMA designed and did the experiments and wrote the manuscript; JDM helped with sample processing and microscopy, TK helped with proliferation and apoptosis experiments, JCB provided VSV $\Delta 51$ virus, and FFL conceived 
the study and obtained funding.All authors read and approved the final manuscript.

\section{Acknowledgments}

This work has been supported by funds from the Canadian Institutes of Health Research, and the Dr. Mariano Elia Chair in Head and Neck Cancer Research. We acknowledge the philanthropic support from the Wharton Family, Joe's Team, and Mr. Gordon Tozer. Dr. NM Alajez is a recipient of a scholarship from the Terry Fox Foundation Strategic Training Initiative for Excellence in Radiation Research for the $21^{\text {st }}$ Century (EIRR21), at the Canadian Institutes of Health Research (CIHR). Support is also provided from the Campbell Family Institute for Cancer Research, and the Ministry of Health and Long-Term Planning.

\section{Author details}

${ }^{1}$ Stem Cell Unit, Department of Anatomy, College of Medicine, King Saud University, Riyadh, Saudi Arabia. ${ }^{2}$ Ontario Cancer Institute, Toronto, ON, Canada. ${ }^{3}$ Department of Medical Biophysics, University of Toronto, Toronto, ON, Canada. ${ }^{4}$ Centre for Cancer Therapeutics, Ottawa Health Research Institute, Ottawa, ON, Canada. ${ }^{5}$ Department of Biochemistry, Microbiology and Immunology, University of Ottawa, Ottawa, ON, Canada. ${ }^{6}$ Department of Radiation Oncology, University Health Network, Toronto, ON, Canada. ${ }^{7}$ Department of Radiation Oncology, University of Toronto, Toronto, ON, Canada. ${ }^{8}$ Department of Radiation Oncology, Princess Margaret Hospital/ Ontario Cancer Institute, 610 University Avenue, Toronto, ON, CanadaM5G $2 \mathrm{Mg}$

Received: 17 March 2012 Accepted: 29 May 2012

Published: 15 June 2012

\section{References}

1. Marur S, Forastiere AA: Head and neck cancer: changing epidemiology, diagnosis, and treatment. Mayo Clin Proc 2008, 83(4):489-501.

2. Giralt J, Benavente S, Arguis M: Optimizing approaches to head and neck cancer: strengths and weaknesses in multidisciplinary treatments of locally advanced disease. Ann Oncol 2008, 19(Suppl 7):vii195-199.

3. Bell JC, Lichty B, Stojdl D: Getting oncolytic virus therapies off the ground. Cancer Cell 2003, 4(1):7-11.

4. Melcher A, Parato K, Rooney CM, Bell JC: Thunder and lightning: immunotherapy and oncolytic viruses collide. Mol Ther, 19(6):1008-1016.

5. Stojdl DF, Lichty BD, tenOever BR, Paterson JM, Power AT, Knowles S, Marius R, Reynard J, Poliquin L, Atkins H, et al: VSV strains with defects in their ability to shutdown innate immunity are potent systemic anti-cancer agents. Cancer Cell 2003, 4(4):263-275.

6. Moussavi M, Fazli L, Tearle H, Guo Y, Cox M, Bell J, Ong C, Jia W, Rennie PS: Oncolysis of prostate cancers induced by vesicular stomatitis virus in PTEN knockout mice. Cancer Res 2010, 70(4):1367-1376.

7. Alain T, Lun X, Martineau Y, Sean P, Pulendran B, Petroulakis E, Zemp FJ, Lemay CG, Roy D, Bell JC, et al: Vesicular stomatitis virus oncolysis is potentiated by impairing mTORC1-dependent type I IFN production. Proc Natl Acad 2010, 107(4):1576-1581.

8. Alajez NM, Mocanu JD, Shi W, Chia MC, Breitbach CJ, Hui AB, Knowles S, Bell JC, Busson P, Takada K, et al: Efficacy of systemically administered mutant vesicular stomatitis virus (VSVDelta51) combined with radiation for nasopharyngeal carcinoma. Clin Cancer Res 2008, 14(15):4891-4897.

9. Blakey DC, Ashton SE, Westwood FR, Walker M, Ryan AJ: ZD6126: a novel small molecule vascular targeting agent. Int J Radiat Oncol Biol Phys 2002 54(5):1497-1502

10. Schepelmann S, Ogilvie LM, Hedley D, Friedlos F, Martin J, Scanlon I, Chen $\mathrm{P}$, Marais $\mathrm{R}$, Springer $\mathrm{CJ}$ : Suicide gene therapy of human colon carcinoma xenografts using an armed oncolytic adenovirus expressing carboxypeptidase G2. Cancer Res 2007, 67(10):4949-4955.

11. Yong RL, Shinojima N, Fueyo J, Gumin J, Vecil GG, Marini FC, Bogler O, Andreeff M, Lang FF: Human bone marrow-derived mesenchymal stem cells for intravascular delivery of oncolytic adenovirus Delta24-RGD to human gliomas. Cancer Res 2009, 69(23):8932-8940.

12. Breitbach CJ, Burke J, Jonker D, Stephenson J, Haas AR, Chow LQ, Nieva J, Hwang TH, Moon A, Patt R, et al: Intravenous delivery of a multi-mechanistic cancer-targeted oncolytic poxvirus in humans. Nature, 7362:99-102.

13. Advani SJ, Markert JM, Sood RF, Samuel S, Gillespie GY, Shao MY, Roizman B, Weichselbaum RR: Increased oncolytic efficacy for high-grade gliomas by optimal integration of ionizing radiation into the replicative cycle of HSV-1. Gene Ther, 11:1098-1102.

14. Gaddy DF, Lyles DS: Oncolytic vesicular stomatitis virus induces apoptosis via signaling through PKR, Fas, and Daxx. J Virol 2007, 81(6):2792-2804.

15. Breitbach CJ, Paterson JM, Lemay CG, Falls TJ, McGuire A, Parato KA, Stojdl DF, Daneshmand M, Speth K, Kirn D, et al: Targeted inflammation during oncolytic virus therapy severely compromises tumor blood flow. Mol Ther 2007, 15(9):1686-1693.

16. Alonso MM, Gomez-Manzano C, Jiang H, Bekele NB, Piao Y, Yung WK, Alemany R, Fueyo J: Combination of the oncolytic adenovirus ICOVIR-5 with chemotherapy provides enhanced anti-glioma effect in vivo. Cancer Gene Ther 2007, 14(8):756-761.

17. Diallo JS, Boeuf FL, Lai F, Cox J, Vaha-Koskela M, Abdelbary H, Mactavish H, Waite K, Falls T, Wang J, et al: A High-throughput Pharmacoviral Approach Identifies Novel Oncolytic Virus Sensitizers. Mol Ther 2010 18(6):1123-1129.

18. Mocanu JD, Yip KW, Alajez NM, Shi W, Li JH, Lunt SJ, Moriyama EH, Wilson BC, Milosevic M, Lo KW, et al: Imaging the modulation of adenoviral kinetics and biodistribution for cancer gene therapy. Mol Ther 2007, 15(5):921-929.

19. Mocanu JD, Yip KW, Skliarenko J, Shi W, Busson P, Lo KW, Bastianutto C, Liu FF: Imaging and modulating antisense microdistribution in solid human xenograft tumor models. Clin Cancer Res 2007, 13(19):5935-5941.

20. Alajez NM, Shi W, Hui AB, Yue S, Ng R, Lo KW, Bastianutto C, O'Sullivan B, Gullane P, Liu FF: Targeted depletion of BMI1 sensitizes tumor cells to P53-mediated apoptosis in response to radiation therapy. Cell Death Differ 2009, 16(11):1469-1479.

21. Alajez NM, Lenarduzzi M, Ito E, Hui AB, Shi W, Bruce J, Yue S, Huang SH, Xu W, Waldron J, et al: miR-218 Suppresses Nasopharyngeal Cancer Progression through Downregulation of Survivin and the SLIT2-ROBO1 Pathway. Cancer Res 2011, 71(6):2381-2391.

22. Trotter MJ, Chaplin DJ, Durand RE, Olive PL: The use of fluorescent probes to identify regions of transient perfusion in murine tumors. Int J Radiat Oncol Biol Phys 1989, 16(4):931-934

doi:10.1186/1475-2867-12-27

Cite this article as: Alajez et al:: Enhanced vesicular stomatitis virus (VSV $\Delta 51$ ) targeting of head and neck cancer in combination with radiation therapy or ZD6126 vascular disrupting agent. Cancer Cell International 2012 12:27.

\section{Submit your next manuscript to BioMed Central and take full advantage of:}

- Convenient online submission

- Thorough peer review

- No space constraints or color figure charges

- Immediate publication on acceptance

- Inclusion in PubMed, CAS, Scopus and Google Scholar

- Research which is freely available for redistribution 